Editorial

\title{
Alternobaric vertigo: Eustachian tube function should be assessed before vestibular function
}

Keywords: alternobaric vertigo, Eustachian tube catheterization, Eustachian tube dysfunction

Abbreviations: ETD, Eustachian tube dysfunction; ABV, alternobaric vertigo

\section{Unilateral Eustachian tube dysfunction was} first known as "alternobaric vertigo" (vertigo alternobarica)

In 1838, Nicolas Deleau, an expert in Eustachian tube catheterization, reported at least one patient who presented with dizziness, tinnitus, and hearing impairment. ${ }^{1}$ These were later defined as the three symptoms of Menière's disease. ${ }^{2}$ Deleau treated the patient by catheterizing the Eustachian tube and administering an air douche, which ameliorated symptoms. ${ }^{1}$ This early case is a typical example of occlusion of the Eustachian tubes resulting in hearing loss, tinnitus, and vertigo, and demonstrates why Eustachian tube dysfunction (ETD) should be ruled out before a diagnosis of Meniere's disease can be made. ${ }^{2}$ In fact, ETD has long been recognized as a principal cause of hearing loss, tinnitus, and vertigo. Therefore, patients exhibiting such symptoms should undergo inflation of the Eustachian tubes as the first step in a thorough clinical investigation. ${ }^{3}$

Over a century after Deleau's first report, in 1942, F.W. Merica ${ }^{3}$ declared that vertigo caused by obstruction of the Eustachian tubes is a distinct clinical entity. Obstruction of the Eustachian tube disturbs the air pressure which stimulates the perilymph and interferes with normal balance which is maintained by the labyrinthine mechanism (Figure 1). Vertigo associated with ETD is caused in most (and perhaps all) instances by unilateral Eustachian tube obstruction or by more complete obstruction one side than the other. In the literature, most references to ETD-related vertigo are made in general discussion on vertigo due to various causes. ${ }^{3}$

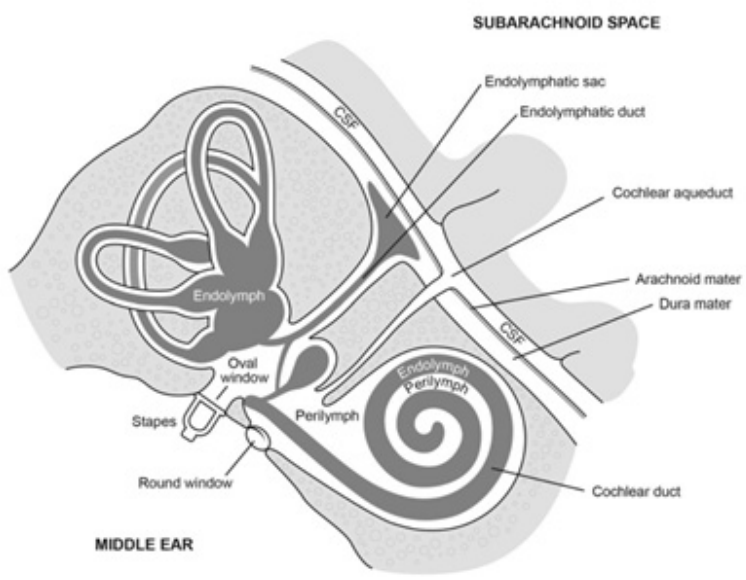

Figure I Demonstration of the communication between cerebrospina fluid (CSF) and perilymph via the cochlear aqueduct. Adapted from "SCUBA Medicine for otolaryngologists: Part I . Diving in to SCUBA physiology and injury prevention.” By Mallen JR, Roberts DS. 2019, Laryngoscope, 9999, 3.
Volume 12 Issue 2 - 2020

\author{
Hee Young Kim \\ Department of Otolaryngology, Kim ENT Clinic, Republic of \\ Korea
}

Correspondence: Hee-Young Kim, Department of Otolaryngology, Kim ENT Clinic, 2nd fl. I 19, Jangseungbaegi-ro Dongjak-gu, Seoul, 06935, Republic of Korea, ORCID 00000002-9400-3098, Tel +82 02855 754I, Fax +82 028557542 Email kheeyoun@gmail.com

Received: March 06, 2020 | Published: March 10, 2020

Vertigo due to unilateral ETD was first defined as "alternobaric vertigo" (or 'vertigo altenobarica'), by Dr. Claes Lundgren ${ }^{4}$ who coined the term in 1965 to describe vertigo in deep-sea divers. ${ }^{4}$ It was also used to describe vertigo in aircraft pilots in $1966 .{ }^{5}$ In both professions, the phenomenon primarily occurs during ascent (and rarely descent) as a result of asymmetrical middle ear pressures (Figure 2). ${ }^{6}$

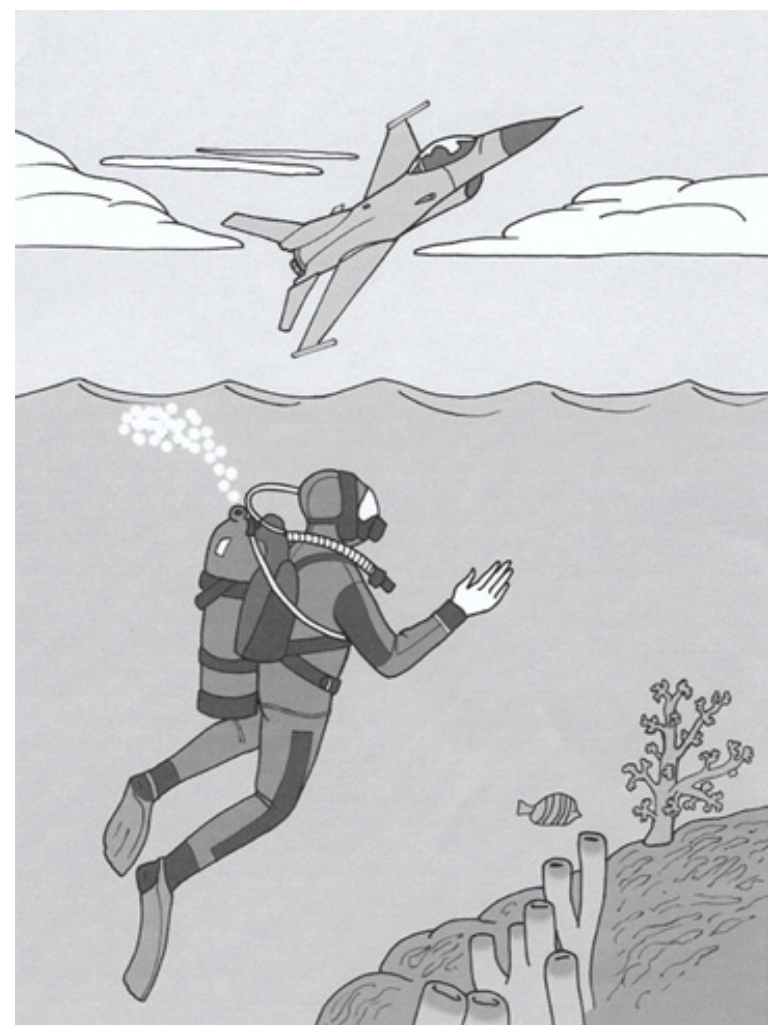

Figure 2 Cartoon depicting that alternobaric vertigo can occur in scuba divers and airplane pilots during ascent.Adapted from "Case Report: Persistent Alternobaric Vertigo at Ground Level due to Chronic Toynbee phenomenon." by Bluestone CD, Swarts JD, Furman JM, et al. 2012, Laryngoscope, 122(4):7. 
Alternobaric vertigo (ABV) results from unequal pressure between the middle ears and is usually caused by the pressures changing at different rates ${ }^{7}$ causing the brain to erroneously perceive the difference as movement. This fundamental mechanism of ABV can be applied to cases of $\mathrm{ABV}$ at ground level with asymmetrical middle ear pressures, no matter how minute the difference in pressure is. ABV may be accompanied by a feeling of fullness, tinnitus, and muffled hearing in one or both ears. ${ }^{78}$ In severe cases, nausea, vomiting, and nystagmus can also occur.

$\mathrm{ABV}$ should be differentiated from any condition conferring active risk of vertigo or severe disequilibrium. This includes peripheral causes such as Menière's disease, benign paroxysmal positional vertigo, and vertebrogenic dizziness, as well as central disorder. ${ }^{9}$

Persistent alternobaric vertigo at ground level shows abnormal vestibular function test results

In 2012, for the first time, Dr. Charles Bluestone ${ }^{5,6}$ published a case report documenting a case of persistent alternobaric vertigo at ground level due to chronic Toynbee phenomenon. Bluestone reported that the pathogenesis of otitis media in such individuals may be related to abnormal anatomy causing ETD. In the reported case, vestibular function was abnormal and Eustachian tube function tests revealed dysfunction of the tube. In order to relieve the Toynbee phenomenon, surgery was performed to replace the obstructed ventilation tube with a patent one. Adenoidectomy and bilateral inferior turbinate reduction were performed to relieve chronic nasal obstruction. Postoperatively, the Eustachian tube remained dysfunctional, however vestibular function returned to normal, and vertigo was completely resolved. Bluestone concluded that nasal obstruction and the Toynbee phenomenon may be involved in patients who have vertigo (Figure $3){ }^{6}$

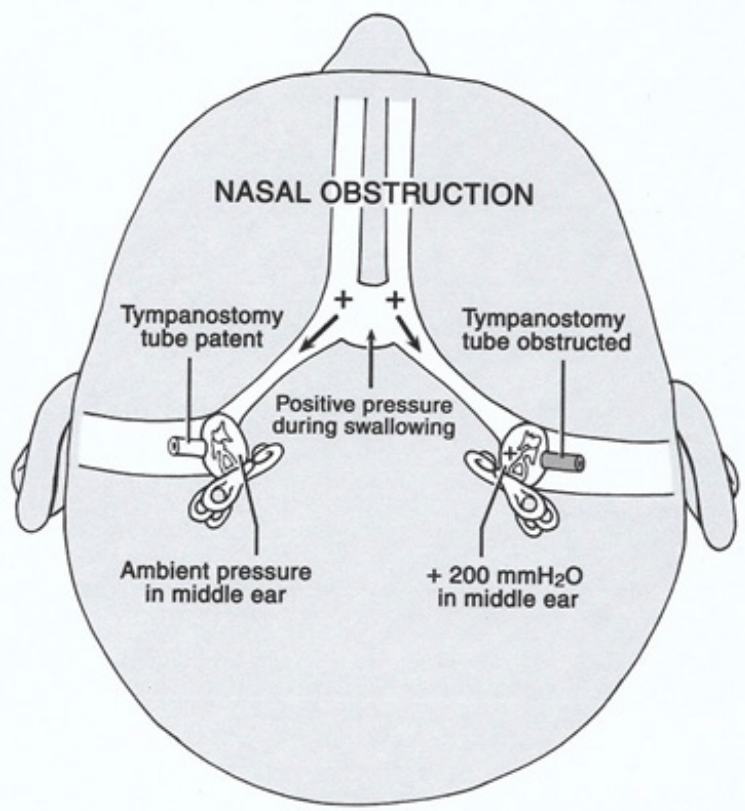

Figure 3 Cartoon showing the pathogenesis of persistent alternobaric vertigo in our patient who had chronic nasal obstruction, the Toynbee Phenomenon, and a unilaterally obstructed tympanostomy tube that created high positive middle-ear pressure. Adapted from "Case Report: Persistent Alternobaric Vertigo at Ground Level due to Chronic Toynbee phenomenon.” by Bluestone CD, Swarts JD, Furman JM, et al. 20I2, Laryngoscope, I22(4):8.
One of the most important reasons for assessing Eustachian tube function is the need to make a differential diagnosis in patients with intact tympanic membrane without evidence of otitis media, but with symptoms potentially related to ETD (otalgia, snapping or popping in the ear, fluctuating hearing loss, tinnitus, or vertigo). ${ }^{5}$ This strongly indicates that ETD must be ruled out before any vestibular function test.

\section{Eustachian tube function should be assessed before vestibular function}

Vestibular organ dysfunction is caused by poorly regulated pressure in the middle ear. Vestibular organs are considered to be dependent variable organs. ${ }^{10}$ Every clinical test currently used to assess vestibular function should ideally be performed in a state where pressure in the middle ear cavity is at the perfectly normal value, and perfectly balanced between the two ears. ${ }^{2,11-13}$

Taking these points into consideration, I would like to call for further case studies and researches into cases of vertigo where Eustachian tube function is assessed before vestibular function is tested. Such investigation may provide exciting insights which impact practice. In addition, publication without consideration of this point, may require revision.

\section{Acknowledgments}

The author has no acknowledgments.

\section{Conflicts of interest}

The author declares that there is no conflict of interest to disclose.

\section{Funding details}

The author has no funding, financial relationship.

\section{References}

1. Mudry A, History of otology. A tribute to Adam Politzer. Wayenborgh: Piribebuy/Paraguay; 2015.

2. Kim HY. The History of Eustachian Tube Catheterization. $J$ Otolaryngology ENT Res. 2016;4(1):00084.

3. Merica FW. Vertigo due to obstruction of the Eustachian tubes. JAMA. 1942;118(15):1282-1284.

4. Lundgren CE. Alternobaric vertigo - a diving hazard. Brit Med J. 1965;2(5460):511-513.

5. Bluestone CD. Eustachian tube: Structure, function, and role in MiddleEar Disease. 2e Kindle ed. People Medical Publishing House USA: Raleigh, North Carolina; 2018.

6. Bluestone CD, Swarts JD, Furman JM, et al. Case Report: Persistent Alternobaric Vertigo at Ground Level due to Chronic Toynbee phenomenon. Laryngoscope. 2012;122(4):868-872.

7. Klingmann $\mathrm{C}$, Knauth M, Praetorius $\mathrm{M}$, et al. Alternobaric vertigo really a hazard? Otol Neurotol. 2006;27(8):1120-1125.

8. Kitajima N, Sugita-Kitajima A, Kitajima S. Altered Eustachian tube function in scuba divers with alternobaric vertigo. Otol Neurotol. 2014;35(5):850-856.

9. Mallen JR, Roberts DS. SCUBA Medicine for Otolaryngologists: Part II. Diagnostic, Treatment, and Dive Fitness Recommendations. Laryngoscope. 2019;9999:1-6.

10. Kim HY. Vertigo due to Eustachian Tube Dysfunction. Archives of Otorhinolaryngology-Head \& Neck Surgery. 2017;1(1):5. 
11. Kim HY. Reciprocal Causal Relationship between Laryngopharyngeal Reflux and Eustachian Tube Obstruction. J Otolaryngol ENT Res. 2015;2(6):00046.

12. Kim HY. Diagnosis \& Treatment of Mechanical Obstruction of Eustachian Tube. J Otolaryngol ENT Res. 2014;1(1):01.
13. Kim HY. Eustachian Tube Catheterization. J Otolaryngol ENT Res. 2015;3(2):00057. 